Lymphology 53 (2020) 99-108

\title{
SKIN LYMPHATIC SYSTEM IN THE PATHOGENESIS OF ARTERIAL HYPERTENSION - REVIEW AND CRITIQUE
}

\author{
A. Chachaj, A. Szulba
}

Department of Angiology, Hypertension and Diabetology, Wroclaw Medical University, Wroclaw, Poland

\section{ABSTRACT}

Although numerous studies have confirmed the relationship between high salt intake and elevated blood pressure, the exact molecular mechanisms of this relationship are still unclear. There is growing evidence that skin interstitium, as well as the skin lymphatic system, are important regulators of both sodium ( $\mathrm{Na}+$ ) balance and blood pressure. Skin is in itself a large reservoir of $\mathrm{Na}+$ ions which are stored in an osmotically inactive form on glycosaminoglycans (GAGs). Local hypertonicity due to extensive accumulation of $\mathrm{Na}+$ within the skin as a result of a high-salt diet was demonstrated to induce macrophages to express a transcription factor termed tonicityresponsive enhancer binding protein (TonEBP) and subsequently to secrete vascular endothelial growth factor-C (VEGF-C), activating lymphangiogenesis within the skin. This regulatory axis seems to be adaptive in maintaining blood pressure in high salt-load states. Recent studies have added new insights into the functioning of lymphatic vessels and the pathogenesis of salt-sensitive hypertension as well as questioned the classic view of $\mathrm{Na}+$ homeostasis. This review aims to summarize recent findings pertaining to the involvement of the skin lymphatic system in $\mathrm{Na}+$ and blood pressure regulation.

Keywords: arterial hypertension, skin lymphatics, lymphangiogenesis, sodium, salt sensitivity, glycosaminoglycans (GAGs), tonicity-responsive enhancer binding protein
(TonEBP), vascular endothelial growth factorC (VEGF-C)

The skin is the largest and complex organ in humans and is made up of two layers (1). The most superficial layer, the epidermis, is avascular. The deeper skin dermis however contains a widely distributed network of both blood and lymphatic vessels. Lymphatic capillaries within the skin originate as the blind-ending initial lymphatics, which begin to converge and coalesce, forming progressively larger collecting lymphatic vessels (2). The skin lymphatic system can be subdivided into superficial and deep lymphatic plexuses. The superficial plexus is formed only by the lymphatic capillary network, whereas the deep lymphatic plexus contains also collecting lymphatic vessels (3) (Fig. 1).

The primary function of the lymphatic system is the maintenance of fluid homeostasis within tissues by providing a route for the return of the extravasated interstitial fluid into the bloodstream. The lymphatic system is also a component of the immune system. Impairment of the dermal lymphatic system results in edema as well as recurrent skin infections (2). Several recent studies have highlighted that the lymphatic system plays an important role in the pathophysiology of various cardiovascular and metabolic diseases, including atherosclerosis, insulin resistance, diabetes, and obesity $(4,5)$. There is also increasing evidence that lymphatic vessels in the skin are crucial in the pathogenesis of salt-sensitive hypertension. 


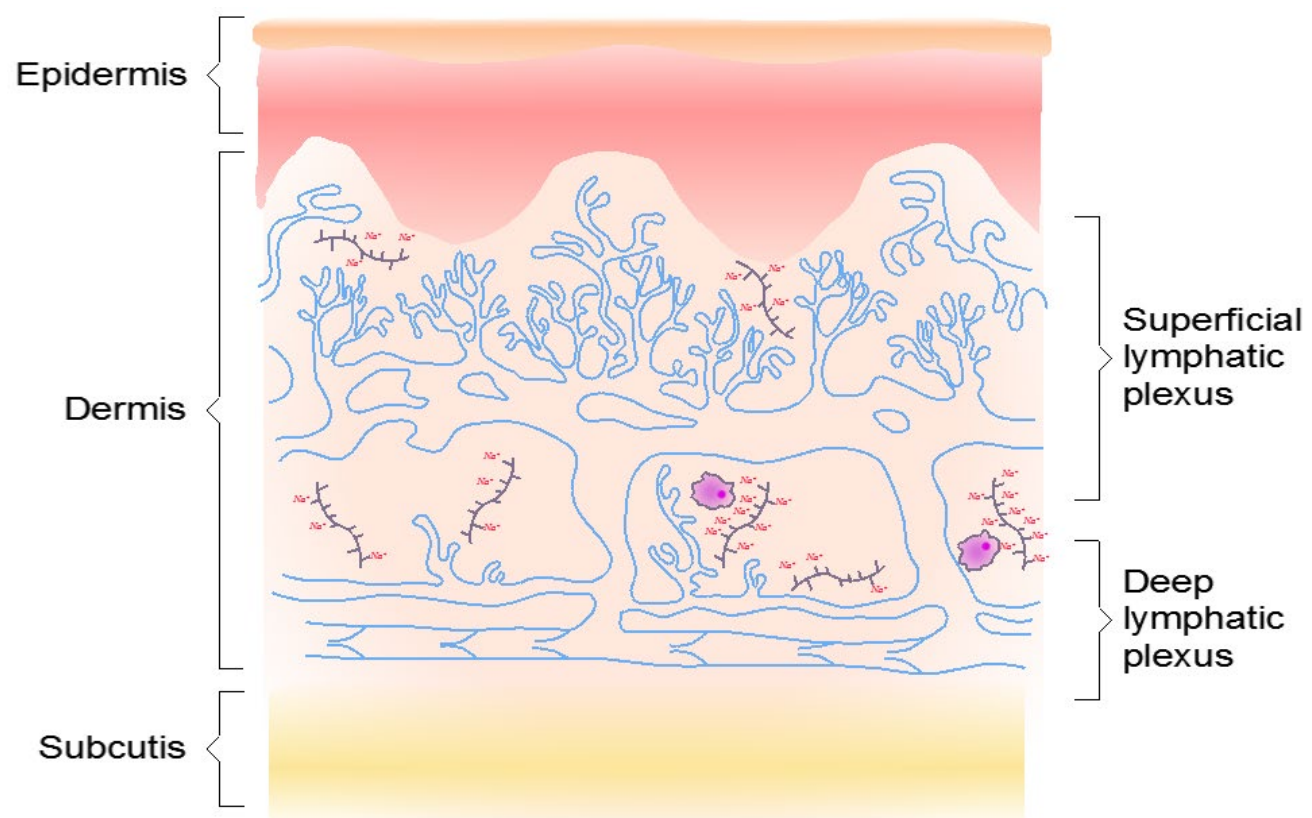

Fig. 1. Schematic of lymphatic vessels, glycosaminoglycans and macrophages in the skin.

The concept that arterial hypertension might result from lymphatic system dysfunction was hypothesized for the first time in 1998 (6). To date, the direct relationship between salt-sensitive hypertension and the skin lymphatic system has been demonstrated mainly within the rodent model $(7,8)$, and many questions still remain unanswered. New discoveries continue to change our traditional understanding of both skin interstitium functioning and $\mathrm{Na}+$ and fluid homeostasis and have opened a new door for future investigations.

\section{Salt Sensitivity}

Numerous clinical and experimental studies have demonstrated that a high-salt diet is associated with the development of hypertension (9-12). Current guidelines recommend reduction of salt intake to $5 \mathrm{~g} /$ day in the general population, and this recommendation is seen as one of the most important steps in the non-pharmacological management of hypertension (13). However, recent data from the PURE study suggests that salt reduction below a certain level $(<3 \mathrm{~g} /$ day) may be harmful and the optimal daily intake of salt should be between 4-6g/day $(14,15)$.

Generally, salt sensitivity is defined as a susceptibility to demonstrate a significant increase in blood pressure following salt loading and a decrease in blood pressure following a reduction in salt intake. Conversely, salt resistance means that blood pressure does not change or may even fall after salt supplementation $(9,16,17)$. Salt-sensitivity occurs in $50 \%$ of patients with hypertension in the Caucasian race and in $75 \%$ of hypertensives among Afro-Americans (18-21). This trait is not only limited to the hypertensive group but also occurs in normotensives (approximately in $20 \%)(20,21)$. It becomes more prevalent in older age $(16,17)$, renal dysfunction (22), obesity, and diabetes mellitus (23). It leads to the development of hypertension and is associated with a worse prognosis (24). When survival curves were examined, normotensive salt-sensitive subjects were found to have cumulative mortality similar to that of hypertensive subjects (24). The precise molecular mechanisms 
underlying the effects of excessive dietary $\mathrm{Na}+$ on high blood pressure are still not clear and many different hypotheses have been proposed. Recent findings suggest that salt sensitivity is associated with vascular dysfunction manifested by the inability of blood vessels to vasodilate in response to salt loading (25). It has also been demonstrated that excessive $\mathrm{Na}+$ intake causes severe damage to the endothelial glycocalyx, which then enables $\mathrm{Na}+$ entry into the endothelial cells and results in endothelial dysfunction (26). Other plausible explanations of vascular dysfunction from salt sensitivity relate to the possible inhibition of vasodilation by asymmetrical dimethylarginine (ADMA), the levels of which are abnormally increased by $\mathrm{Na}+(27)$. Another proposed explanation of salt sensitivity pertains to a possible interaction between the immune system and the vasculature, resulting in an inflammatory state at sites of excessive accumulation of $\mathrm{Na}+(25,28,29)$. In line with these studies is the finding that a high-salt diet significantly increases hormonal vasoreactivity of pre-capillary resistance arterioles in the skin toward angiotensin-2 and noradrenaline, and this way could increase peripheral resistance and contribute to higher blood pressure in salt-sensitive hypertension (30).

\section{Three-Compartment Model of Sodium Balance}

Numerous sodium balance studies have indicated that exposure to a high-salt diet can lead to $\mathrm{Na}+$ retention as the excessive $\mathrm{Na}+$ load is not completely excreted by the kidneys but accumulates without commensurate water retention elsewhere in the body (three-compartments model of sodium balance) (31-34). Interestingly, human spaceflight studies have further supported this osmotically inactive $\mathrm{Na}+$ storage mechanism (35). Long-term observations in subjects with a constant $\mathrm{Na}+$ intake have shown large fluctuations in diurnal $\mathrm{Na}+$ excretion, with no correlation between $\mathrm{Na}+$ intake and excretion. There was however a correlation between $\mathrm{Na}+$ excretion and aldosterone or cortisol secretion, and these correlations were independent of blood pressure value or body water content (36).

\section{Sodium and Glycosaminoglycans in the Skin Interstitium}

Ivanova et al as early as in $\mathbf{1 9 7 8}$ demonstrated that $\mathrm{Na}+$ can accumulate within the skin of rats and that under conditions of high $\mathrm{Na}+$ intake the concentration of skin $\mathrm{Na}+$ increases. It was suggested that the mechanism behind this accumulation of $\mathrm{Na}+$ was an increase in $\mathrm{Na}+$ binding to sulphated glycosaminoglycans (GAGs) within the skin $(37,38)$. Further experiments on the rodent model performed by Titze and collaborators confirmed that large amounts of $\mathrm{Na}+$ are stored in the skin (39-42). In the study of Heer et al, $\mathrm{Na}+$ retention in the skin was also confirmed to exist in healthy men (32). We examined $\mathrm{Na}+$ concentrations within abdominal skin specimens taken during elective surgery in patients both in hypertensive and control groups. Although we demonstrated that $\mathrm{Na}+$ storage is present in human skin, we found no statistical difference in skin $\mathrm{Na}+$ concentrations between the study groups. Moreover, we observed that increased $\mathrm{Na}+$ content was associated with higher water retention (43). Studies with ${ }^{23} \mathrm{Na}$ magnetic resonance imaging $\left({ }^{23} \mathrm{Na}-\mathrm{MRI}\right)$ enable quantitative visualization of $\mathrm{Na}+$ content within the body (44). ${ }^{23} \mathrm{Na}$-MRI studies have shown that $\mathrm{Na}+$ storage in humans occurs primarily within the skin and skeletal muscle without concomitant water retention (45). ${ }^{23} \mathrm{Na}$-MRI also revealed that $\mathrm{Na}+$ content within the skin increases with age $(45,46)$, is higher in men (45), in hypertension (45), and in other conditions which are known to be associated with salt sensitivity, including type 2 diabetes (47) and in hemodialysis patients (46).

Titze et al also reported that $\mathrm{Na}+$ is stored within the skin of rodents in an osmotically inactive form attached to interstitial GAGs $(7,39)$. GAGs are linear polysaccharide chains which possess a significant negative charge density (48) and have been shown to respond to increased dermal $\mathrm{Na}+$ content by further increasing their charge density through increased polymerization and sulfonation $(7,32,39)$. Conversely, long-term salt deprivation in rats appears to result in the 
mobilization of osmotically inactive dermal $\mathrm{Na}+$ from GAGs, and the concentration of GAGs decreases (49). A recent study in mice demonstrated that defective heparin sulfate polymerization leads to a decrease in $\mathrm{Na}+$ and water content within the skin as well as to a higher heart rate, increased fluid intake, increased plasma osmolality, and to an abnormal response to $\mathrm{Na}+$ loading (50). These strongly support the theory that GAGs within the dermal interstitium enable the skin to manage bound $\mathrm{Na}+$ stores and to regulate water homeostasis (50).

Macrophages - TonEBP - VEGF-C Lymphangiogenesis Axis in Salt-Sensitive Hypertension

Machnik et al demonstrated that rats fed a high-salt diet develop an increase in tonicity and osmotic stress within the dermal interstitium due to extensive skin $\mathrm{Na}+$ storage. Hypertonic electrolyte accumulation within the skin promotes the infiltration of the mononuclear phagocyte system (MPS) cells $(7,8)$. Macrophages are mobile osmosensors which actively move to the sites of higher salt concentration in the skin. Chemotaxis toward sites with higher salt concentration has been shown to be a macrophage-specific process although the exact molecular mechanism requires further investigation (51).

It has also been shown that MPS cells express a transcription factor termed tonicityresponsive enhancer binding protein (TonEBP, also known as nuclear factor of activated T cells 5, NFAT5), which subsequently increases the secretion of vascular endothelial growth factor C (VEGF-C), a known major lymphangiogenic growth factor. VEGF-C secreted by MPS cells acts via the VEGF receptor (VEGFR)-3 and stimulates lymphangiogenesis and increases lymphatic vessel density within the skin (8). VEGF-C also induces endothelial nitric oxide synthase (eNOS) expression (52). The impact of VEGF$\mathrm{C}$ on lymphatic capillary vessels in the skin of rats on high-salt diet was manifest by both increased lymphatic capillary quantity as well as an increase in lymphatic capillary diameter, reflecting both lymphangiogenesis as well as hyperplasia of the preexisting lymph capillary network in response to dietary salt loading (8). Further studies have confirmed the functional nature of newly formed lymphatics induced by salt accumulation within the skin $(53,54)$. Inhibition of the TonEBP - VEGF-C VEGFR3 axis in mice may be accomplished through genetic deletion of the TonEBP gene in MPS cells, by selective pharmacological blockade of the VEGFR3 receptor with an antibody, or by skin-specific trapping of VEGF-C in the skin. It results in the prevention of lymphangiogenesis, accumulation of electrolytes within the skin, and in elevated blood pressure (8). Similarly, macrophage depletion or inhibition of VEGF-C signaling within the skin of rats fed a high-salt diet promotes or aggravates salt-sensitive hypertension (55). These findings confirm that the lymphatic system likely plays an important role in the regulation of blood pressure and are in agreement with the clinical observations that hemangiogenesis/lymphangiogenesis inhibitors, which block VEGF signaling pathways and which are used in anticancer therapy, often result in hypertension $(56,57)$.

The increased number and volume of dermal lymphatic capillaries resulting from continued lymphangiogenesis, may represent an adaptive mechanism that allows for a more effective form of removal of both $\mathrm{Na}+$ and water from the skin interstitium $(8,55)$. According to this view, the lymphatic system probably ensures that any $\mathrm{Na}+$ stored within the skin is able to return to the blood circulation and then be excreted mainly via the urine. A high-salt diet has been demonstrated to enhance skin and muscle lymph flow by up to $26 \%$ and by $20 \%$, respectively, as well as to increase the frequency of lymphatic vessel contractions (53). A high-salt diet has also been shown to differentially modulate the mechanical activity of afferent and efferent collecting lymphatics in murine iliac lymph nodes (58). These studies indicate that the lymphatic system adjusts its structure and activity to the quantity of $\mathrm{Na}+$ within the skin. The osmolality and electrolyte concentration in lymph draining skin are equal in rats fed a 
high-salt diet in comparison to rats fed a lowsalt diet (59), suggesting that the clearance of osmolytes and electrolytes from tissues occurs via enhanced fluid clearance (53). This suggestion points to the importance of lymph flow as an integrated element of electrolyte homeostasis (53). Na+ removal from the skin might decrease the influence of $\mathrm{Na}+$ accumulation on blood vessel constriction and, as a consequence, might lower blood pressure (30). Increased skin VEGF-C upregulates eNOS and NO production resulting in vasodilation. This mechanism may also directly contribute to blood pressure reduction (52) as it has been demonstrated that dermal vasculature contains biologically significant stores of NO which has the potential to influence systemic blood pressure (60).

\section{VEGF-C and Salt-Sensitive Hypertension}

The role of VEGF-C does not appear to be limited to its being the main mediator of lymphangiogenesis as well as an inductor of eNOS expression $(7,8)$. It was also been shown that VEGF-C demonstrates a potential effect on the contractility of lymphatic collectors in the skin with higher $\mathrm{Na}+$ concentrations, probably through VEGFR-3 (61). VEGF-C promotes the synthesis of hyaluronic acid resulting in a higher charge density of synthesized GAGs within the endothelial glycocalyx (62). Thus, it is possible that VEGF-C could also affect the actual structure of the GAGs within the skin, but this will require further investigation.

Concentrations of serum VEGF-C in hypertensive patients compared to control groups have been inconsistent in different studies - in some studies showing higher concentrations (8) while others, including our study, demonstrated lower concentrations $(43,46)$. The concentration of circulating VEGF-C however appears to decrease with age (46). Interestingly, $\mathrm{Na}+$ content within the skin increases with age. Thus, it has been suggested that the lower levels of VEGF-C in older age could be associated with lower $\mathrm{Na}+$ clearance and could, therefore, lead to both a greater $\mathrm{Na}+$ accumulation within the skin as well as potential arterial hypertension (46). Furthermore, dialysis patients with higher VEGF-C levels have been shown to be significantly better at $\mathrm{Na}$ + clearance from the skin reservoirs during hemodialysis treatment (46). It has also been demonstrated that VEGF-C concentrations were higher during a high-salt diet than during a low-salt diet in patients with chronic kidney disease, whereas in healthy subjects this relationship was not statistically relevant $(63,64)$. The concentra-tion of circulating VEGF-C might, therefore, be the marker of salt-sensitivity, as it has been demonstrated that circulating VEGF-C increases during a high-salt diet in saltsensitive, but not salt-resistant, subjects (64).

\section{Immune System and Lymphatic Vessels in Salt-sensitive Hypertension}

Osmotic stress MPS cells infiltrate the skin interstitium in response to osmotic stress, with the induction of the TonEBP - VEGF-C VEGFR3 regulatory axis, but without concomitant TNF $\lambda$ (tumor necrosis factor $\lambda$ ) gene or protein expression (55). It has, therefore, been suggested that lymphangiogenesis in the skin, as a result of an excessive storage of $\mathrm{Na}+$ within the skin, proceeds without a concomitant inflammatory response component (55). Nevertheless, there is growing evidence that the relationship between skin $\mathrm{Na}+$ storage, the immune system, lymphatic vessels, and hypertension is strong and complex $(65,66)$.

Chronic inflammation stimulates lymphangiogenesis (67). The role of T lymphocytes in the pathogenesis of salt-sensitive hypertension has been recently well established $(68,69)$. Salt has been shown to promote the differentiation of CD4+ T cells into a Th-17 pro-inflammatory phenotype, which results in vascular dysfunction (70). Moreover, recent studies suggest that dendritic cells (DCs), which migrate from the skin to skin-draining lymph nodes, also may have a role in lymphangiogenesis within the skin interstitium. Such an assumption was based on the observation that although DCs within lymph nodes in the non-inflammatory steadystate mostly do not originate from upstream 
tissue DCs, after an inflammatory stimulus, lymph-migrating tissue-derived DCs make up the majority of lymph node DCs (71). Further studies are needed to determine the exact role of dermal DCs in the process of lymphangiogenesis and $\mathrm{Na}+$ homeostasis (65).

More studies are also necessary to determine the exact mechanisms regarding the participation of macrophages in the pathophysiology of hypertension. Although the role of macrophages in the skin seems to be protec-tive against salt-sensitive hypertension as macrophage depletion results in salt-sensitive hypertension in rats fed a high-salt diet (55), subsequent studies have suggested far more complex mechanisms concerning macrophages that need to be further clarified. Firstly, macrophages are highly heterogeneous immune cells and exhibit two different activation states - M1 (pro-inflammatory) and M2 (anti-inflammatory) (72). It has been demonstrated in rats that hypertension is associated with the M1 phenotype of macrophage and not with M2 (73). Moreover, it has also been revealed that, in the presence of noninflammatory signals (e.g., IL-4 and IL-13), salt reduces macrophage activation while, conversely, salt appears to augment macrophage activation in a pro-inflammatory environment (e.g., lipopolysaccharide, LPS). This suggests that salt does not have one definite impact on macrophages but is modulated by other stimuli $(\mathbf{7 4 , 7 5 )}$.

\section{Regulation of $\mathrm{Na}+$ Accumulation in the Skin Interstitium}

The molecular mechanisms of $\mathrm{Na}+$ entering the skin interstitium and its subsequent mobilization from interstitial tissue are still largely unknown. Studies with the use of ${ }^{23} \mathrm{Na}$ MRI have demonstrated that dialysis (46), spironolactone (46) and dapagliflozin (76) can mobilize $\mathrm{Na}+$ from both the skin and muscle in humans and have the potential to decrease tissue content of $\mathrm{Na}+$.

Recent findings also suggest that there is a local electrolyte gradient in the skin interstitium and that the skin creates its own electrolyte microenvironment. In the study of
Nikpey et al, it was demonstrated in rats that there is an osmolite gradient from epidermis through dermis to subcutis. This study also demonstrated that lymph draining the skin is isosmotic to plasma. Earlier studies pertaining to this aspect were inconclusive (59). The presence of an osmolite gradient within the skin had already been previously demonstrated in a study using $x$-rays of the human skin (77). Moreover, in the 7T 23Na MRI study, it was shown that there is a greater accumulation of $\mathrm{Na}+$ directly beneath the epidermis in comparison to the deeper layers of the dermis (78). Taking into account that greater $\mathrm{Na}+$ accumulation occurred directly beneath the epidermis but was not associated with water accumulation, it has been hypothesized that the skin may contain a kidney-like countercurrent system with active transport of $\mathrm{Na}+$ within the skin and with mobile macrophages acting as $\mathrm{Na}+$ sensors. These would then have the potential to regulate lymphatic function in $\mathrm{Na}$ + clearance via the TonEBP - VEGF-C VEGFR3 axis $(65,79,80)$. Further physiological studies are needed to confirm such presumptions.

\section{CONCLUSIONS}

The skin lymphatic system seems to be an integrated element of electrolyte and water homeostasis and plays a key role in the pathogenesis of hypertension. This new knowledge might be essential for the development of novel therapeutic options for hypertensive patients, especially those with salt-sensitive hypertension.

\section{CONFLICT OF INTEREST AND DISCLOSURE}

The authors declare no competing financial interests exist.

\section{REFERENCES}

1. Nguyen, AV, AM Soulika: The dynamics of the skin's immune system. Int. J. Mol. Sci. 20 (2019), 1811.

2. Szuba A, SG Rockson: Lymphedema: 
Anatomy, physiology and pathogenesis. Vasc. Med. 2 (1997), 321-326.

3. Petrova, TV, GY Koh: Organ-specific lymphatic vasculature: From development to pathophysiology. J. Exp. Med. 215 (2018), 35-49.

4. Aspelund, A, MR Robciuc, S Karaman, T Makinen, K Alitalo: Lymphatic system in cardiovascular medicine. Circ. Res. 118 (2016), 515-530.

5. Jiang, X, W Tian, MR Nicolls, SG Rockson: The lymphatic system in obesity, insulin resistance, and cardiovascular diseases. Front Physiol. 10 (2019), 1402.

6. Mekarski, JE: Essential hypertension is lymphatic: A working hypothesis. Med. Hypotheses 51 (1998), 101-103.

7. Titze, J, A Machnik: Sodium sensing in the interstitium and relationship to hypertension. Curr. Opin. Nephrol. Hypertens. 19 (2010), 385-392.

8. Machnik, A, W Neuhofer, J Jantsch, et al: Macrophages regulate salt-dependent volume and blood pressure by a vascular endothelial growth factor-C-dependent buffering mechanism. Nat. Med. 15 (2009), 545-552.

9. Morimoto, A, T Uzu, T Fujii, et al: Sodium sensitivity and cardiovascular events in patients with essential hypertension. Lancet 350 (1997), 1734-1737.

10. Intersalt: An international study of electrolyte excretion and blood pressure. Results for 24 hour urinary sodium and potassium excretion. Intersalt Cooperative Research Group. BMJ 297 (1988), 319-328.

11. Geleijnse, JM, JC Witteman, A Hofman, DE Grobbee: Electrolytes are associated with blood pressure at old age: The Rotterdam Study. J Hum Hypertens 11 (1997), 421-423.

12. Forte, JG, JM Miguel, MJ Miguel, F de Padua, G Rose: Salt and blood pressure: A community trial. J. Hum. Hypertens. 3 (1989), 179-184.

13. Kjeldsen, SE, K Narkiewicz, M Burnier, $\mathrm{S}$ Oparil: 2018 Practice guidelines for the management of arterial hypertension of the European Society of Hypertension. Blood Press 27 (2018), 313.

14. Mente, A, M O'Donnell, S Rangarajan, et al: Associations of urinary sodium excretion with cardiovascular events in individuals with and without hypertension: A pooled analysis of data from four studies. Lancet 388 (2016), 465-475.

15. O'Donnell, M, A Mente, S Rangarajan, et al: Urinary sodium and potassium excretion, mortality, and cardiovascular events. N. Engl. J. Med. 371 (2014), 612-623.

16. Weinberger, MH, JZ Miller, FC Luft, CE
Grim, NS Fineberg: Definitions

andcharacteristics of sodium sensitivity and blood pressure resistance. Hypertension 8 (1986), II127-34.

17. Kawasaki, T, CS Delea, FC Bartter, H Smith: The effect of high-sodium and low-sodium intakes on blood pressure and other related variables in human subjects with idiopathic hypertension. Am. J. Med. 64 (1978), 193-198.

18. Sullivan, JM, RL Prewitt, TE Ratts: Sodium sensitivity in normotensive and borderline hypertensive humans. Am. J. Med. Sci. 295 (1988), 370-377.

19. Luft, FC, JZ Miller, CE Grim, et al: Salt sensitivity and resistance of blood pressure. Age and race as factors in physiological responses. Hypertension 17 (1991), I102-108.

20. Sullivan, JM, TE Ratts, JC Taylor, et al: Hemodynamic effects of dietary sodium in man: a preliminary report. Hypertension 2 (1980), 506-514.

21. Weinberger, MH: Dietary sodium and blood pressure. Hosp. Pract. (Off Ed) 21 (1986), 55-64.

22. Weir, MR, JC Fink: Salt intake and progression of chronic kidney disease: An overlooked modifiable exposure? A commentary. Am. J. Kidney Dis 45 (2005), 176-188.

23. Tuck, ML: Role of salt in the control of blood pressure in obesity and diabetes mellitus. Hypertension 17 (1991), I135-142.

24. Weinberger, MH, NS Fineberg, SE Fineberg, M Weinberger: Salt sensitivity, pulse pressure, and death in normal and hypertensive humans. Hypertension 37 (2001), 429-432.

25. Laffer, CL, RC Scott, 3rd, JM Titze, FC Luft, F Elijovich: Hemodynamics and salt-andwater balance link sodium storage and vascular dysfunction in salt-sensitive subjects. Hypertension 68 (2016), 195-203.

26. Oberleithner, H, W Peters, K Kusche-Vihrog, et al: Salt overload damages the glycocalyx sodium barrier of vascular endothelium. Pflugers Arch. 462 (2011), 519-528.

27. Schmidlin, O, A Forman, A Leone, A Sebastian, RC Morris, Jr: Salt sensitivity in blacks: Evidence that the initial pressor effect of $\mathrm{NaCl}$ involves inhibition of vasodilatation by asymmetrical dimethylarginine. Hypertension 58 (2011), 380-385.

28. Mattson, DL: Infiltrating immune cells in the kidney in salt-sensitive hypertension and renal injury. Am. J. Physiol. Renal Physiol. 307 (2014), F499-508. 
29. Mathis, KW, HJ Broome, MJ Ryan: Autoimmunity: an underlying factor in the pathogenesis of hypertension. Curr Hypertens Rep 16 (2014), 424.

30. Helle, F, TV Karlsen, O Tenstad, J Titze, H Wiig: High-salt diet increases hormonal sensitivity in skin pre-capillary resistance vessels. Acta Physiol. (Oxf) 207 (2013), 577-581.

31. Heer, M, F Baisch, J Kropp, R Gerzer, C Drummer: High dietary sodium chloride consumption may not induce body fluid retention in humans. Am. J. Physiol. Renal Physiol. 278 (2000), F585-595.

32. Heer, M, P Frings-Meuthen, J Titze, et al: Increasing sodium intake from a previous low or high intake affects water. Br. J. Nutr. 101 (2009), 1286-1294.

33. Palacios, C, K Wigertz, BR Martin, et al: Sodium retention in black and white female adolescents in response to salt intake. J. Clin. Endocrinol. Metab. 89 (2004), 1858-1863.

34. Titze J: Water-free $\mathrm{Na}$ + retention: Interaction with hypertension and tissue hydration. Blood Purif. 26 (2008), 95-99.

35. Gerzer, R, M Heer: Regulation of body fluid and salt homeostasis--from observations in space to new concepts on earth. Curr. Pharm. Biotechnol. 6 (2005), 299-304.

36. Rakova, N, K Juttner, A Dahlmann, et al: Long-term space flight simulation reveals infradian rhythmicity in human $\mathrm{Na}(+)$ balance. Cell Metab. 17 (2013), 125-131.

37. Ivanova, LN, VK Archibasova, I Shterental: [Sodium-depositing function of the skin in white rats]. Fiziol. Zh. SSSR Im. I. M. Sechenova 64 (1978), 358-363.

38. Arkhibasova, VI, LN Ivanova, GV Podsekaeva, V Natochin Iu: [Possibilities of deposition of electrolytes in the skin in different states of water-salt metabolism]. Fiziol. Zh. SSSR Im. I. M. Sechenova 69 (1983), 1352-1356.

39. Titze, J, R Lang, C Ilies, et al: Osmotically inactive skin $\mathrm{Na}$ + storage in rats. Am. J. Physiol. Renal Physiol 285 (2003), F1108-1117.

40. Titze, J, K Bauer, M Schafflhuber, et al: Internal sodium balance in DOCA-salt rats: A body composition study. Am. J. Physiol. Renal Physiol, 289 (2005), F793-802.

41. Ziomber, A, A Machnik, A Dahlmann, et al: Sodium-, potassium-, chloride-, and bicarbonate-related effects on blood pressure and electrolyte homeostasis in deoxycorticosterone acetate-treated rats. Am. J. Physiol. Renal Physiol. 295 (2008), F1752-1763.

42. Titze, J, M Shakibaei, M Schafflhuber, et al: Glycosaminoglycan polymerization may enable osmotically inactive $\mathrm{Na}+$ storage in the skin. Am. J. Physiol. Heart Circ. Physiol. 287 (2004), H203-208.

43. Chachaj, A, B Pula, M Chabowski, et al: Role of the lymphatic system in the pathogenesis of hypertension in humans. Lymphat. Res. Biol. 16 (2018), 140-146.

44. Kopp, C, P Linz, L Wachsmuth, et al: (23)Na magnetic resonance imaging of tissue sodium. Hypertension 59 (2012), 167-172.

45. Kopp, C, P Linz, A Dahlmann, et al: $23 \mathrm{Na}$ magnetic resonance imaging-determined tissue sodium in healthy subjects and hypertensive patients. Hypertension 61 (2013), 635-640.

46. Dahlmann, A, K Dorfelt, F Eicher, et al: Magnetic resonance-determined sodium removal from tissue stores in hemodialysis patients. Kidney Int. 87 (2015), 434-441.

47. Schneider, MP, U Raff, C Kopp, et al: Skin sodium concentration correlates with left

48. Wiig, H, FC Luft, JM Titze: The interstitium conducts extrarenal storage of sodium and represents a third compartment essential for extracellular volume and blood pressure homeostasis. Acta Physiol. (Oxf) 222 (2018), doi: 10.1111/apha.13006.

49. Schafflhuber, M, N Volpi, A Dahlmann, et al: Mobilization of osmotically inactive $\mathrm{Na}+$ by growth and by dietary salt restriction in rats. Am. J. Physiol Renal Physiol. 292 (2007), F1490-1500.

50. Olde Engberink, RHG, J de Vos, A van Weert, et al: Abnormal sodium and water homeostasis in mice with defective heparan sulfate polymerization. PLoS One 14 (2019), e0220333.

51. Muller, S, T Quast, A Schroder, et al: Saltdependent chemotaxis of macrophages. PLoS One 8 (2013), e73439.

52. Lahdenranta, J, J Hagendoorn, TP Padera, et al: Endothelial nitric oxide synthase mediates lymphangiogenesis and lymphatic metastasis. Cancer Res. 69 (2009), 2801-2808.

53. Karlsen, TV, E Nikpey, J Han, et al: Highsalt diet causes expansion of the lymphatic network and increased lymph flow in skin and muscle of rats. Arterioscler. Thromb. Vasc. Biol. 38 (2018), 2054-2064.

54. Kwon, S, GD Agollah, W Chan, EM SevickMuraca: Altered lymphatic function and architecture in salt-induced hypertension assessed by near-infrared fluorescence imaging. J. Biomed. Opt. 17 (2012), 080504-1.

55. Machnik, A, A Dahlmann, C Kopp, et al: Mononuclear phagocyte system depletion 
blocks interstitial tonicity-responsive enhancer binding protein/vascular endothelial growth factor $\mathrm{C}$ expression and induces salt-sensitive hypertension in rats. Hypertension 55 (2010), 755-761.

56. Baek Moller, N, C Budolfsen, D Grimm, et al: Drug-induced hypertension caused by multikinase inhibitors (Sorafenib, Sunitinib, Lenvatinib and Axitinib) in renal cell carcinoma treatment. Int. J. Mol. Sci 20 (2019), 4712.

57. Wu, S, JJ Chen, A Kudelka, J Lu, X Zhu: Incidence and risk of hypertension with sorafenib in patients with cancer: A systematic review and meta-analysis. Lancet Oncol. 9 (2008), 117-123.

58. Mizuno, R, M Isshiki, N Ono, M Nishimoto, T Fujita: A high-salt diet differentially modulates mechanical activity of afferent and efferent collecting lymphatics in murine iliac lymph nodes. Lymphat. Res. Biol. 13 (2015), 85-92.

59. Nikpey, E, TV Karlsen, N Rakova, et al: High-salt diet causes osmotic gradients and hyperosmolality in skin without affecting interstitial fluid and lymph. Hypertension 69 (2017), 660-668.

60. Mowbray, M, S McLintock, R Weerakoon, et al: Enzyme-independent NO stores in human skin: Quantification and influence of UV radiation. J. Invest. Dermatol. 129 (2009), 834-842.

61. Mizuno, R, M Isshiki, N Ono, M Nishimoto, T Fujita: A high salt diet alters pressureinduced mechanical activity of the rat lymphatics with enhancement of myogenic characteristics. Lymphat. Res. Biol. 13 (2015), 2-9.

62. Foster, RR, L Armstrong, $S$ Baker, et al: Glycosaminoglycan regulation by VEGFA and VEGFC of the glomerular microvascular endothelial cell glycocalyx in vitro. Am. J. Pathol. 183 (2013), 604-616.

63. Slagman, MC, AJ Kwakernaak, S Yazdani, et al: Vascular endothelial growth factor $\mathrm{C}$ levels are modulated by dietary salt intake in proteinuric chronic kidney disease patients and in healthy subjects. Nephrol. Dial Transplant. (2011), 978-982.

64. Liu, F, J Mu, Z Yuan, et al: Involvement of the lymphatic system in salt-sensitive hypertension in humans. Med. Sci. Monit. 17 (2011), CR542-546.

65. Oh, YS, LJ Appel, ZS Galis, et al: National Heart, Lung, and Blood Institute Working
Group Report on Salt in Human Health and Sickness: Building on the Current Scientific Evidence. Hypertension 68 (2016), 281-288.

66. Harrison, DG, PJ Marvar, JM Titze: Vascular inflammatory cells in hypertension. Front Physiol. 3 (2012), 128.

67. Varricchi, G, F Granata, S Loffredo, A Genovese, G Marone: Angiogenesis and lymphangiogenesis in inflammatory skin disorders. J. Am. Acad. Dermatol. 73 (2015), 144-153.

68. Ren, J, SD Crowley: Role of T-cell activation in salt-sensitive hypertension. Am. J. Physiol. Heart Circ. Physiol. 316 (2019), H1345-H1353.

69. Wenzel, UO, M Bode, C Kurts, H Ehmke: Salt, inflammation, IL-17 and hypertension. Br. J. Pharmacol. 176 (2019), 1853-1863.

70. Binger, KJ, RA Linker, DN Muller, M Kleinewietfeld: Sodium chloride, SGK1, and Th17 activation. Pflugers Arch. 467 (2015), 543-550.

71. Jakubzick, C, M Bogunovic, AJ Bonito, et al: Lymph-migrating, tissue-derived dendritic cells are minor constituents within steady-state lymph nodes. J. Exp. Med. 205 (2008), 2839-2850.

72. Xue, J, SV Schmidt, J Sander, et al: Transcriptome-based network analysis reveals a spectrum model of human macrophage activation. Immunity 40 (2014), 274-288.

73. Ndisang, JF, M Mishra: The heme oxygenase system selectively suppresses the proinflammatory macrophage $\mathrm{m} 1$ phenotype and potentiates insulin signaling in spontaneously hypertensive rats. Am. J. Hypertens. 26 (2013), 1123-1131.

74. Binger, KJ, M Gebhardt, $M$ Heinig, et al: High salt reduces the activation of IL-4- and IL-13stimulated macrophages. J. Clin. Invest. 125 (2015), 4223-4238.

75. Jantsch, J, V Schatz, D Friedrich, et al: Cutaneous $\mathrm{Na}+$ storage strengthens the antimicrobial barrier function of the skin and boosts macrophage-driven host defense. Cell Metab. 21 (2015), 493-501.

76. Karg, MV, A Bosch, D Kannenkeril, et al: SGLT-2-inhibition with dapagliflozin reduces tissue sodium content: A randomised controlled trial. Cardiovasc. Diabetol. 17 (2018), 5.

77. Warner, RR, MC Myers, DA Taylor: Electron probe analysis of human skin: Element concentration profiles. J. Invest. Dermatol. 90 (1988), 78-85. 
78. Linz, P, D Santoro, W Renz, et al: Skin sodium measured with (2)(3)Na MRI at 7.0

T. NMR Biomed. 28 (2015), 54-62.

79. Titze, J: A different view on sodium balance. Curr. Opin. Nephrol. Hypertens. 24 (2015), 14-20.

80. Hofmeister, LH, S Perisic, J Titze: Tissue sodium storage: evidence for kidney-like extrarenal countercurrent systems? Pflugers Arch. 467 (2015), 551-558.
Andrzej Szuba, MD, PhD

Professor of Medicine

Wroclaw Medical University

Department of Angiology

Borowska 213 Street

50-556 Wroclaw, Poland

Phone/Fax: +48 717332200

E-mail: szubaa@yahoo.com andrzej.szuba@umed.wroc.pl 